\title{
Lifetime Broadening of a Nuclear Magnetic Resonance Peak under Minimal Length Uncertainty Analysis
}

\author{
A. FARMANY* \\ Physics Department, Azad University of Ilam, Iran \\ and \\ Michigan Center for Theoretical Physics, Michigan University, MI, United States \\ (Received November 3, 2009; revised version February 11, 2010; in final form March 5, 2010)
}

\begin{abstract}
We calculate the lifetime broadening of a nuclear magnetic resonance peak based on the minimal length uncertainty. The application of high resolution nuclear magnetic resonance spectroscopy and its extension to detection of the signature of minimal length scale is explored. We show that in a high precision spectrometry test, the lifetime broadening is a quadratic function with energy.
\end{abstract}

PACS numbers: 11.30.Er, 11.25.Sq, 11.15.-q

\section{Introduction}

It is expected that some low energy relics of high energy phenomena may be verified by nowadays experiments, and some phenomenological low energy effects may be explored in solvable models at a level of quantum mechanics in a minimal length scale [1-9]. In literature, the minimal length quantum mechanics have been extensively studied $[10,1,2]$. In this letter we study the possibility of testing minimal length scale via nuclear magnetic resonance spectroscopy taking into account the solution of broadening of a magnetic resonance peak by minimal length uncertainty. One manifestation of the uncertainty principle is the effect of decreasing the lifetime of a nucleus in a given environment on the nuclear magnetic resonance spectrum of the same nucleus or one to which it is coupled. These kinetic effects may have experimental significance by giving rise to spectra whose shift-state values or line widths may be used as a measure, or to spectra which are simplified so that the resonances are more suitable for quantitative measurements. The shifting and merging of spectra by kinetic process may also be used to shift an increasing resonance away from a resonance of interest if the interfering species can be made to undergo rapid exchange.

The magnitude of the effect can be estimated from uncertainty principle, $\Delta E \Delta t \geq \hbar$. Since $\Delta E=\hbar \Delta \nu$ and $\Delta t$ may be identified with $\tau$, the average lifetime of the excited state in the various exchanging states, the relationship becomes $\tau \Delta \nu \approx 1 / 2 \pi$. Thus the magnitude of the rate constant that will give an observable effect will vary for different types of spectrometry, depending on the frequency difference involved. The widest application of this principle has been in nuclear magnetic resonance spectrometry, especially proton NMR. When a system

* e-mail: a.farmany@usa.com under observation by NMR is undergoing slow change between different environments, separate resonances are observed for each environment. At higher rates of exchange, the resonances are broadened and shifted toward each other, and with fast exchange, a single resonance is observed at a position which is a weighted average of the positions and relative population of various environments. In order to all effects that cause to broadening of peaks, there is a new effect that acts as a constraint on the broadening of peaks, this effect rises from the existence of a minimal length uncertainty [3].

\section{Theory}

Let line broadening is due to the lifetime broadening, since absorption of the radiation $\mathrm{aL}(\mathrm{w})$ ays produces an excited state with a finite lifetime. What is the relation between lifetime and broadening of spectra? To obtain the answer we describe the kinetic decay of an excited state using an exponential function. This emerges directly from the first order kinetics. Looking ahead, this means that if we define the rate of disappearance of the excited state $E$ as

$$
\frac{\partial[E]}{\partial t}=-\frac{[E]}{T_{2}}
$$

Eq. (1) could be solved analytically as

$$
E(t)=E_{0} \mathrm{e}^{-t / T_{2}}
$$

where $T_{2}$ is the excited state lifetime. Conjugated energy width can be obtained from the Fourier transformation of the lifetime function,

$$
L(w)=\sqrt{\frac{2}{\pi}} \int_{0}^{\infty} \exp \left(-\left(\frac{1}{T_{2}}+\mathrm{i} w\right) t\right) \mathrm{d} t .
$$

Solution of Eq. (3) reads

$$
L(w)=\sqrt{\frac{2}{\pi}} \frac{1}{T_{2}^{-1}-\mathrm{i} w},
$$

which is a complex function. The imaginary and real 
parts of Eq. (4) may be solved by multiplying both numerator and denominator by $T_{2}^{-1}+\mathrm{i} w$ that gives

$$
L w=\sqrt{\frac{2}{\pi}}\left[\frac{T_{2}^{-1}}{\left(T_{2}^{-1}\right)^{2}+w^{2}}+\frac{\mathrm{i} w}{\left(T_{2}^{-1}\right)^{2}+w^{2}}\right] .
$$

The real part of this integral is a Lorentzian line shape function. This line shape will be observed for transitions that have only homogeneous broadening. Let us note that we have used $T_{2}$ as the relaxation time; this brings up the important analogy between optical spectroscopy and NMR spectroscopy. The relaxation time $T_{2}$ has two components $T_{1}$ and $T_{2}^{*}$ as the lifetime of state or longitudinal relaxation time and transverse relaxation time, respectively. The lifetime contributes to the relaxation time by decreasing the population of the excited state. Pure de-phasing contributes to the $T_{2}$ relaxation time by destroying coherence between the ground and excited states

$$
\Lambda(w)=\frac{T_{2}^{-1}}{\pi\left(\left(T_{2}^{-1}\right)^{2}+w^{2}\right)} .
$$

It is interesting to obtain the relation between the relaxation time and the line width. The Lorentzian that we have discussing so far is centered about zero frequency. The maximum is

$$
\Lambda(0)=\frac{T_{2}}{\pi} .
$$

At the half-maximum we obtain the highest as

$$
\frac{\Lambda_{0}}{2}=\frac{T_{2}}{2 \pi}
$$

and the width at half-maximum is determined by solving

$$
\frac{T_{2}^{-1}}{\pi\left(\left(T_{2}^{-1}\right)^{2}+w^{2}\right)}=\frac{T_{2}}{2 \pi} \text {. }
$$

Solution of (9) yields

$$
w= \pm T_{2}^{-1} \text {. }
$$

The width $\Gamma$ is known as the full-width at half-maximum (FWHM). It is easy to see that $\Gamma=2 / T_{2}$, which corresponds to a homogeneous Lorentzian line width, where $T_{2}=2 T_{1}$ and $\Gamma=1 / T_{1}$. As an important and well founded result, the line width is related to the excited state lifetime. Using the uncertainty principle $\Delta E T_{1} \approx \hbar$ we can write

$$
T_{1} \Gamma \approx 1, \Delta E=\hbar \Gamma,
$$

where $T_{1}$ alone is responsible of relaxation. Let us consider the effect of minimal length uncertainty on Eq. (11). An exciting quantum mechanical implication of the microphysics space is a modification of the uncertainty principle as

$$
\Delta x \geq \frac{\hbar}{\Delta p}+\alpha^{\prime} \frac{\Delta p}{\hbar},
$$

where $\sqrt{\alpha^{\prime}}$ is the Planck length. From Eq. (12) it is easy to obtain a similar relation between time and energy as (for details see [3]):

$$
\Delta t \geq \frac{1}{\Delta E}+t^{\prime} \Delta E
$$

Let us note that in (13) we have used the natural units $\alpha^{\prime}, c, \hbar=1$. Combining (11) with (13) and substituting $\Delta t$ with $T_{1}$ we obtain

$$
\Gamma^{-1} \geq \frac{1}{\Delta E}+t^{\prime} \Delta E
$$

and

$$
t^{\prime} \Delta E^{2}-\Gamma^{-1} \Delta E+1 \approx 0,
$$

Eq. (15) can be solved as

$$
\Delta E_{\min } \approx \frac{\Gamma}{2 t^{\prime}}\left(1-\sqrt{1-\frac{4 t^{\prime}}{\Gamma^{2}}}\right) .
$$

Expanding (16) around $t^{\prime}=0$, we obtain,

$$
\Delta E_{\min } \approx \Gamma+t^{\prime}\left(\Gamma^{3}\right)+\ldots
$$

Comparing Eq. (17) with Eq. (11), it is easy to see that the first term of the right hand side of Eq. (17) is the standard line width (note that in (17) we have used a system in which ) of an excited state and the other terms are the signature of the minimal length uncertainty on the lifetime broadening of a NMR peak.

\section{Summary}

The above consideration is generalized the nuclear magnetic resonance spectroscopy for a NMR test experiment with a high precision experiment. The typical size of this correction in an actual system is $10^{-3} \%$.

\section{Acknowledgements}

I would like to thank the referee for helpful comments.

\section{References}

[1] G. Veneziano, Europhys. Lett. 2, 199 (1986); D.J. Gross, P.F. Mende, Nucl. Phys. 303, 407 (1988); K. Konishi, G. Paffuti, P. Provero, Phys. Lett. B 234, 276 (1990); A. Kempf, G. Mangano, R.B. Mann, Phys. Rev. D 52, 1108 (1995); T. Matsuo, Y. Shibusa, Mod. Phys. Lett. A 21, 1285 (2006); Physics 234, 276 (2006); M. Maggiore, Phys. Lett. B 30465 (1993); M. Maggiore, Phys. Rev. D 49, 5182 (1994); T. Matsuo, Y. Shibusa, Mod. Phys. Lett. A 21, 1285 (2006).

[2] Jang Young Bang, M.S. Berger, Phys. Rev. D 74, 125012 (2006); A. Farmany, Electron. J. Theor. Phys. 3, 12 (2004); M. Maggiore, Phys. Lett. B 319, 83 (1993); H.F. Hofmann, Phys. Rev. A 67, 022106 (2003); S. Capozziello, G. Lambiase, G. Scarpetta, Int. J. Theor. Phys. 39, 15 (2000).

[3] A. Farmany, Sh. Abbasi, A. Naghipour, Phys. Rev. D 650, 33 (2007); ibid. 659, 9 (2008), Erratum; A. Farmany, Sh. Abbasi, A. Naghipour, Chaos Solitons Fractals 42, 1097 (2009).

[4] F. Brau, J. Phys. A 32, 7691 (1999).

[5] R. Akhoury, Y.P. Yao, hep-th/03021108.

[6] A. Kempf, J. Phys. A 30, 2093 (1997) and references therein. 
[7] Th. Udem, A. Huber, B. Gross, J. Reichert, M. Prevedelli, M. Weitz, T.W. Hänsch, Phys. Rev. Lett. 79, 2646 (1997).

[8] S. Hossenfelder, M. Bleicher, S. Hofmann, J. Ruppert, S. Scherer, H. Stöcker, Phys. Lett. B 575, 85 (2003).

[9] A. Kempf, G. Managano, Phys. Rev. D 55, 7909 (1997).
[10] S. Benczik, L.N. Chang, D. Minic, T. Takeuchi, Phys. Rev. A 72, 012104 (2005); M.M. Stetsko, V.M. Tkachuk, arXiv:0710.5088; M.M. Stetsko, Phys. Rev. A 74, 062105 (2006). 